\title{
Análise do desenvolvimento motor e qualidade do ambiente domiciliar de crianças pré-escolares
}

\author{
Juliana Falcão Padilha ${ }^{1}$ Enio Júnior Seidel ${ }^{2}$ Fernando Copetti ${ }^{3}$
}

\begin{abstract}
RESUMO
Objetivo - Analisar o desenvolvimento motor e a qualidade do ambiente domiciliar de crianças. Métodos - estudo descritivo, quantitativo, realizado com 44 crianças de 18 a 42 meses, de instituições de ensino infantil particulares da região Centro de Santa Maria-RS. Para avaliar 0 ambiente domiciliar e o desenvolvimento motor utilizou-se respectivamente: Affordance in the Home Environment for Motor Development-Self Report e Peabody Developmental Motor Scales-2. Resultado - A média de idade foi 31,6 meses; 59,09\% eram do sexo feminino e $40,91 \%$ masculino; $50 \%$ residem em apartamento; $72,72 \%$ dos lares proporcionam oportunidades suficientes para 0 desenvolvimento motor. Os sub-testes que apresentaram melhor e pior desempenho motor foram respectivamente: manipulação fina e locomoção. Em relação ao Cociente Motor Total, $63,64 \%$ das crianças encontram-se com escore médio. Conclusão - 0 desenvolvimento motor da maioria das crianças avaliadas encontra-se dentro do padrão de normalidade para a idade cronológica, e as residências avaliadas possibilitam oportunidades razoáveis para o desenvolvimento motor.
\end{abstract}

Descritores: Desenvolvimento Infantil; Ambiente Sociocultural; Habilidades Motoras; Crianças Pré-Escolares.

\section{Analysis of motor development and environmental quality household of preschool children}

\begin{abstract}
Objective - The aim of this study was to analyze the motor development and quality of the household environment of children. Methods - This is a descriptive and quantitative study, conducted with 44 children between 18-42 months of private educational institutions for children in the Central Santa Maria, RS. Affordance in the Home Environment for Motor Development - Self Report and Peabody Developmental Motor Scales-2 were used to evaluate the household environment and motor development. Results - The main age was 31.6 months, $59.09 \%$ were female and $40.91 \%$ male, $50 \%$ live in apartment, $72.72 \%$ of households provide sufficient opportunities for motor development. The sub-tests that showed the best and worst motor performance were: fine manipulation and locomotion. Regarding the Total Motor Quotient, $63.64 \%$ are children with an average score. Conclusion - The motor development of most of the children lies within the normal range for chronological age, the residences allow reasonable opportunities for motor development.
\end{abstract}

Descriptors: Child Development; Sociocultural Environment; Motor Abilities; Child Preschool.

\footnotetext{
${ }^{1}$ Mestranda em Fisioterapia na Universidade do Estado de Santa Catarina (UDESC), Florianópolis, SC, Brasil

2 Doutor em Estatística e experimentação agropecuária pela Universidade Federal de Lavras (UFLA), Lavras, RS, Brasil.

${ }^{3}$ Doutor em Ciência do movimento humano pela Universidade Federal de Santa Maria (UFSM), Santa Maria, RS, Brasil.
} 


\section{Introdução}

O desenvolvimento infantil é um processo que envolve múltiplos aspectos, sendo parte deste processo o crescimento físico, a maturação neurológica, construção de habilidades no que se refere ao comportamento, cognição, afetividade e o social da criança ${ }^{1}$. Sabe-se, portanto, que o desenvolvimento infantil é influenciado por fator genético, fator ambiental e exposição a riscos biológicos ${ }^{2,3,4}$.

O desenvolvimento motor ocorre em um contexto social específico, onde o ambiente em que a criança se desenvolve é importante. Alguns fatores são: a sociedade em que ela vive; a escola; a qualidade das condições de vida; o tamanho da família; interações entre irmãos; e globais circunstâncias socioeconômicas 4 .

A estrutura física das residências compostas por espaços interiores e exteriores constitui-se como um dos primeiros meios de experiência nos anos iniciais de vida da criança ${ }^{5}$. No entanto, a mobília, os brinquedos, e a atenção prestada pelos cuidadores, configuram os affordances no lar com repercussões no desenvolvimento motor infantil ${ }^{6}$. Além disso, o nível socioeconômico pode ser, ou não ${ }^{7}$, um fator interveniente ${ }^{8}$.

0 ambiente domiciliar tem sido apontado como o fator extrínseco que mais influencia o desenvolvimento infantil ${ }^{6}$, de maneira favorável como facilitador, ou desfavorável, o que lentifica o ritmo de desenvolvimento ${ }^{9}$. Desta forma, os cuidadores devem proporcionar um ambiente propício e ideal, visto que o contexto familiar é um dos mais importantes, principalmente nos primeiros anos de vida ${ }^{10}$.

O diagnóstico do desenvolvimento motor permite aos profissionais identificarem os atrasos, o que possibilita a tomada de decisão sobre que habilidades motoras devem ser enfatizadas, bem como, as metas de desempenho da criança $a^{11,12}$. Desta forma, instrumentos de avaliação padronizados têm sido cada vez mais utilizados como auxiliares na avaliação de diferentes aspectos do desenvolvimento motor infantil ${ }^{13}$. Assim, além de detectar precocemente possíveis alterações no desenvolvimento motor em seus diferentes contextos, necessita-se também alertar os responsáveis para que haja a possibilidade de verificar se o ambiente em que vivem é sugestivo, ideal, propício para o bom desenvolvimento motor. Dentro deste contexto, o objetivo desta pesquisa foi analisar o desenvolvimento motor e a qualidade do ambiente domiciliar de crianças com 18 a 42 meses de idade que frequentam Instituições de Ensino Infantil no município de Santa Maria-RS.

\section{Metodologia}

Trata-se de um estudo descritivo, de caráter quantitativo ${ }^{14}$. 0 grupo alvo desta pesquisa foram crianças entre 18 e 42 meses de idade matriculadas nas instituições de ensino infantil (IEI) particulares da região centro de Santa Maria-RS. Foi escolhida a região central da cidade, de maneira intencional, pois é de fácil acesso e localiza-se o maior número de IEI particulares, conforme informação da Secretaria Municipal de Educação da cidade. Foram contatadas todas as 16 IEI, das quais nove aceitaram participar do estudo.

Nas nove IEI encontraram-se 250 crianças aptas a participarem da pesquisa, respeitando os critérios de inclusão do estudo (crianças de ambos os sexos com idade entre 18 e 42 meses) e os de exclusão (presença de lesão neurológica ou alteração genética relatada pelos responsáveis ou pela coordenadora da IEI). Destas, 73 tiveram o consentimento dos pais para a participação no estudo, 29 foram excluídas por não terem realizado toda a bateria dos testes motores, resultando 44 crianças participantes do estudo, 26 meninas e 18 meninos. Os pais e/ou responsáveis assinaram o Termo de Consentimento Livre e Esclarecido (TCLE) e pesquisa foi aprovada pelo Comitê de Ética e Pesquisa (0340.0.243.00010), da Universidade Federal de Santa Maria (UFSM).

Para a avaliação do ambiente doméstico foi utilizado o instrumento Affordance in the Home Environment for Motor Development Self Report (AHEMD-SR) ${ }^{6}$, traduzido e adaptado às condições socioculturais brasileiras, com apoio do Laboratório de Pesquisa em Desenvolvimento Neuromotor da Universidade Metodista de Piracicaba. O projeto AHEMD foi desenvolvido pelo Instituto Politécnico de Viana do Castelo, em Portugal, em conjunto com o Laboratório de Desenvolvimento Motor da Texas A\&M University - EUA. O AHEMD-SR é um instrumento validado que avalia de forma simples, rápida e eficaz as oportunidades (affordances) presentes no contexto do ambiente domiciliar para 0 desenvolvimento motor de crianças entre 18 e 42 meses de idade ${ }^{15}$. Na presente pesquisa este questionário foi respondido pelos responsáveis da criança. Utilizou-se o mesmo critério empregado pelo grupo responsável pela validação da pontuação do AHEMD-SR* . O questionário possui 67 questões divididas em três dimensões: espaço físico da residência; 
atividades diárias; brinquedos e materiais existentes na habitação. $O$ escore de uma dimensão é calculado pela soma dos pontos obtidos para todas as questões dentro de cada dimensão. A pontuação total do instrumento (escore total) é obtida pela soma dos escores das três dimensões. A pontuação máxima para cada questionário é de 20 pontos, e essa remete a uma classificação: baixo, menor que 9 pontos; médio, entre 10 a 16 pontos; e alto, de 17 a 20 pontos.

As variáveis analisadas do questionário AHEMD-SR foram: AHEMD total; espaço exterior; espaço interior; variedade de estimulação; materiais de motricidade fina; materiais de motricidade grossa. Estas variáveis apresentam seus valores estandartizados variando de 0 a 4 pontos, os quais são classificados da seguinte forma: 1 ponto, muito fraco; 2 pontos, fraco; 3 pontos, bom; e 4 pontos, muito bom.

Para avaliar o desenvolvimento motor dos sujeitos utilizou-se o Peabody Developmental Motor Scales 2 (PDMS-2). O PDMS-2 é um instrumento padronizado que avalia a execução das habilidades motoras grossas e finas de crianças até os 71 meses de idade. $O$ instrumento permite a avaliação da competência motora, identificar déficits e desequilíbrios entre os componentes motores finos e grossos, avaliar o progresso da criança, dentre outros. Para isso, ele é dividido em dois componentes motores, os quais se ramificam em seis sub-testes. Quatro destes, relacionados com as habilidades motoras grossas - reflexos, posturais, locomoção e manipulação de objetos; e dois, com as habilidades motoras finas - manipulação fina e integração visuo-motora ${ }^{16}$. O somatório das habilidades motoras grossas remete ao quociente motor grosso (QMG), o somatório das habilidades motoras finas, quociente motor fino (QMF), e a soma dos dois resulta no quociente motor total (QMT). Cada sub-teste é composto por tarefas motoras adequadas à idade do indivíduo e é distribuído em uma sequência crescente de dificuldade. A pontuação é descrita e distribuída de acordo com a tabela 1.

Cada criança foi avaliada individualmente nas dependências da IEI por uma equipe de avaliadores que passaram pelo processo de capacitação para a aplicação do instrumento. Este instrumento destaca-se sendo um dos mais recentes no âmbito da avaliação do desenvolvimento motor infantil ${ }^{17}$.

\begin{tabular}{c|c|c}
\hline \begin{tabular}{c} 
Tabela 1 - Guia para interpretação de valores estandartizados dos sub-testes e dos quocientes motores. \\
\hline $\begin{array}{c}\text { Valor estandarizado } \\
\text { Sub teste }\end{array}$
\end{tabular} & $\begin{array}{c}\text { Valor Estandarizado } \\
\text { Quocientes }\end{array}$ \\
\hline $17-20$ & Muito superior & $131-165$ \\
\hline $15-16$ & Superior & $121-130$ \\
\hline $13-14$ & Acima da média & $111-130$ \\
\hline $8-12$ & Média & $90-110$ \\
\hline $6-7$ & Abaixo da média & $80-89$ \\
\hline $4-5$ & Inferior & $70-79$ \\
\hline $1-3$ & Muito inferior & $35-69$ \\
\hline
\end{tabular}

Fonte: Adaptado de Folio e Fewell $(2000)^{16}$.

Para as análises estatísticas utilizou-se o Statistical Package for the Social Sciences SPSS ${ }^{\circledR}$, versão 14.0 para Windows. Através do teste de Shapiro-Wilk verificou-se que as variáveis não apresentaram normalidade $(p<0,05)$, indicando que devem ser utilizados procedimentos estatísticos não-paramétricos. Foi realizada a análise descritiva das variáveis e a construção de tabelas de distribuições de frequências absolutas e percentuais. Para avaliar as correlações entre variáveis da qualidade do ambiente (affordance) e do desempenho motor, foi utilizada a Análise de Correlação de Spearman ${ }^{18}$. Para comparar o efeito de diferentes categorias de variáveis do ambiente domiciliar sobre o desenvolvimento motor e 0 affordance total foram utilizados os testes de Mann-Whitney e de Kruskall-Wallis ${ }^{18}$. 0 nível de significância utilizado nos testes foi de $5 \%$.

\section{Resultados e discussão}

A média de idade das 44 crianças avaliadas foi de 31,6 meses, variando de 19 meses a 42 meses. Na tabela 2 está apresentada a caracterização dos sujeitos quanto ao sexo, tipo de moradia, tempo de residência na moradia e renda familiar. Observa-se que mais da metade dos sujeitos são do sexo feminino e que metade das crianças moram em casa. No que diz respeito ao tempo habitacional, $77,27 \%$ mora na mesma residência há mais de 1 ano e a maioria das famílias tem renda que vai de $R \$ 2.500,00$ a $R \$ 5.000,00$ ou mais reais mensais. 
Tabela 2 - Distribuição de frequências das variáveis categóricas do AHEMD-SR.

\begin{tabular}{|c|c|c|}
\hline Variável & $\mathrm{N}$ & $\%$ \\
\hline \multicolumn{3}{|l|}{ Sexo } \\
\hline Feminino & 26 & 59,09 \\
\hline Masculino & 18 & 40,91 \\
\hline \multicolumn{3}{|l|}{ Moradia } \\
\hline Apartamento & 22 & 50,00 \\
\hline Casa & 22 & 50,00 \\
\hline \multicolumn{3}{|l|}{ Tempo habitacional } \\
\hline Menos de 6 meses & 4 & 9,09 \\
\hline Entre e e 12 meses & 6 & 13,64 \\
\hline Mais de 12 meses & 34 & 77,27 \\
\hline \multicolumn{3}{|l|}{ Renda familiar (R\$) } \\
\hline Até $2.500,00$ & 15 & 34.09 \\
\hline Entre $2.501,00$ a $4.999,00$ & 16 & 36,36 \\
\hline Mais de $5.000,00$ & 13 & 29,55 \\
\hline
\end{tabular}

AHEMD-SR: Affordance in the Home Environment for Motor Development Self Report.

A tabela 3 mostra a análise descritiva das variáveis do AHEMD-SR nas três dimensões do questionário: espaço físico da residência, atividades diárias e brinquedos e materiais existentes na habitação. Pode-se observar predomínio para o espaço exterior com classificação boa e muito boa. Outro ponto que deve ser destacado é em relação ao espaço interior, onde nenhuma residência obteve escore muito fraco ou fraco, e 93,18\% dos lares possuem ambientes com ótimas oportunidades para o desenvolvimento motor. Mais da metade dos lares, para materiais de motricidade fina e grossa, oferecem poucas oportunidades para o desenvolvimento motor.

Tabela 3 - Distribuição de frequência das variáveis do AHEMD-SR.

\begin{tabular}{|c|c|c|c|c|c|}
\hline \multirow[b]{2}{*}{ Variável } & \multirow{2}{*}{$\begin{array}{l}\text { Muito fraco } \\
\mathrm{N} \quad(\%)\end{array}$} & Fraco & \multicolumn{2}{|r|}{ Bom } & Muito Bom \\
\hline & & $\mathrm{N} \quad(\%)$ & $\mathrm{N}$ & $(\%)$ & $\mathrm{N} \quad(\%)$ \\
\hline Espaço exterior & $10(22,73)$ & $8(18,18)$ & 13 & $(29,55)$ & $13(29,55)$ \\
\hline Espaço interior & - & - & 3 & $(6,82)$ & $41(93,18)$ \\
\hline Variedade de estimulação & $10(22,73)$ & $11(25,00)$ & 6 & $(13,64)$ & $17(38,64)$ \\
\hline Materiais de Motricidade fina & $13(29,55)$ & $16(36,36)$ & 11 & $(25,00)$ & $4 \quad(9,09)$ \\
\hline $\begin{array}{l}\text { Materiais de motricidade grossa } \\
\text { HEMD-SR: Affordance in the Hom }\end{array}$ & $\begin{array}{l}13(29,55) \\
\text { Fnvironmen }\end{array}$ & $15(34,09)$ & & $(18,18)$ & $8(18,18)$ \\
\hline
\end{tabular}

Em relação ao espaço exterior, $59,1 \%$ dos indivíduos encontram-se com lares que oferecem oportunidades ótimas e suficientes para o desenvolvimento motor, ou seja, com escores que vão de bom a muito bom. Estes resultados diferem dos encontrados em pesquisa19, onde foi aplicado o instrumento AHEMD-SR com 128 tutores responsáveis pelas crianças, no município de Juazeiro-Ceará, em que 75\% dos lares com espaço exterior fraco ou muito fraco. Já no que diz respeito ao espaço interior, na presente pesquisa, a maioria das residências, 93,18\%, classificam-se como muito boas, assim, os lares proporcionam ótimas oportunidades de desenvolvimento. Estes resultados vão ao encontro do mesmo estudo supracitado, o qual observou que, também, a maioria da sua amostra (66\%) apresentou espaço interior bom ou muito bom ${ }^{19}$.

Em um estudo ${ }^{20} \mathrm{o}$ qual avaliou o ambiente domiciliar de 21 crianças, com idade entre 18 a 42 meses, frequentadoras de uma creche municipal, na cidade de Passo Fundo, RS, com a utilização do instrumento AHEMD-SR, observou que todos os lares eram desfavoráveis para materiais de motricidade fina e, aproximadamente $95 \%$ eram desfavoráveis para materiais de motricidade grossa. O que difere do presente estudo, onde se obteve $31,2 \%$ e $33,4 \%$ dos lares 
com classificação de bom a muito bom para materiais de motricidade fina e materiais de motricidade grossa, respectivamente.

No presente estudo, a variedade de estimulação dos lares pesquisados mostra que $52,28 \%$ deles classificaram-se como bom ou muito bom, o que oferece suficientes e ótimas oportunidades para o desenvolvimento motor. De forma semelhante, em outros estudos, $42 \%$ e $42,9 \%$ dos lares encontravam-se com escores de bom a muito bom 19,20. Contudo, resultado semelhante foi obtido em uma pesquisa ${ }^{21}$, com 34 crianças frequentadoras de IEl comunitárias de Porto Alegre, RS, na qual $53 \%$ dos casos ofereciam condições favoráveis para o fator variedade de estimulação, tendo escores de bom a muito bom.

Em relação aos valores do AHEMD total, $13,63 \%$ dos lares apresentaram escore baixo, ou seja, os lares apresentam poucas oportunidades de desenvolvimento motor. Já as residências que oferecem oportunidades suficientes, classificadas como médias, totalizam $72,72 \%$. Por fim, $13,63 \%$ obtiveram escore alto, os quais oferecem ótimas oportunidades. A média dos resultados do AHEMD total foi de 12,88, cujo menor resultado atingido foi de 8 pontos e o maior foi 18 pontos.

Ao investigar características ambientais de 52 bebês de 6 a 18 meses de creches públicas e particulares da cidade de Erechim, RS, foi observado que $80 \%$ da amostra apresentou AHEMD total médio ${ }^{22}$, se assemelhando ao resultado da presente pesquisa, onde a maioria dos lares, $72,72 \%$, apresenta AHEMD total médio, significando que os domicílios apresentam condições razoáveis para o desenvolvimento. Estudo5, realizado na cidade de Ahvaz no Irã, com 350 lares, apresentou resultado bastante parecido para este fator, onde o valor médio de AHEMD total encontrado foi de 12,55 que o classifica como médio.

A tabela 4 mostra a análise descritiva das variáveis dos sub-testes e dos quocientes motores do PDMS-2. Em relação aos sub-testes, a maioria das crianças apresentou desempenho médio, ou seja, normal para a idade cronológica. 0 sub-teste que apresentou melhor desempenho motor foi o de manipulação fina, apesar de, conforme a tabela 3 , as residências proporcionarem poucas oportunidades de desenvolvimento motor para materiais de motricidade fina. Já 0 que apresentou pior resultado motor foi o sub-teste de locomoção. Já o sub-teste que obteve maior número de sujeitos com classificação média foi o de integração visuo-motor. Em relação ao QMT, mais da metade dos sujeitos $(63,64 \%)$ encontra-se com escore médio.

Tabela 4 - Distribuição de frequência das variáveis motoras avaliadas no PDMS-2.

\begin{tabular}{|c|c|c|c|c|c|c|c|}
\hline & Muito inferior & Inferior & Abaixo da média & Média & Acima da Média & Superior & Muito superior \\
\hline Variável & $\mathrm{N} \quad(\%)$ & $\mathrm{N} \quad(\%)$ & $\mathrm{N}(\%)$ & $\mathrm{N} \quad(\%)$ & $\mathrm{N}(\%)$ & $\mathrm{N} \quad(\%)$ & $\mathrm{N} \quad(\%)$ \\
\hline Posturais & - & - & $3(6,82)$ & $32 \quad(72,73)$ & $5 \quad(11,36)$ & $3(6,82)$ & $1 \quad(2,27)$ \\
\hline Locomoção & - & $3 \quad(6,82)$ & $4 \quad(9,09)$ & $32 \quad(72,73)$ & $4 \quad(9,09)$ & $1(2,27)$ & - \\
\hline Manipulação de objeto & - & - & $2 \quad(6,82)$ & $31 \quad(70,45)$ & $7 \quad(15,91)$ & $4 \quad(9,09)$ & - \\
\hline Manipulação fina & - & - & - & $30 \quad(68,18)$ & $8(18,18)$ & $6(13,64)$ & - \\
\hline Integração visuo-motor & - & - & $3(6,82)$ & $36 \quad(81,82)$ & $5(11,36)$ & - & - \\
\hline QMF & - & - & $2(4,55)$ & $29 \quad(65,91)$ & $9(20,45)$ & $4 \quad(9,09)$ & - \\
\hline QMG & - & - & $7 \quad(15,91)$ & $26 \quad(59,09)$ & $10(22,73)$ & $1(2,27)$ & - \\
\hline QMT & - & - & $3(6,82)$ & $28 \quad(63,64)$ & $12(27,27)$ & $1(2,27)$ & - \\
\hline
\end{tabular}

QMF: Quociente motor fino; QMG: quociente motor grosso; QMT: Quociente motor total. PDMS-2: Peabody Developmental Motor Scales 2 .

Ao avaliar o desenvolvimento neuropsicomotor de 960 pré-escolares na educação infantil, com idade entre 4 a 6 anos incompletos, matriculados em 27 IEI da cidade de Cuiabá, MT, através do teste de triagem de Denver II, constataram que a maioria $(67 \%)$ dos pré-escolares obteve desempenho normal ${ }^{23}$. Apesar de não ter utilizado o mesmo instrumento, esse resultado é semelhante ao obtido no presente estudo, que observou $63,64 \%$ dos sujeitos com desenvolvimento motor normal (médio).

Na tabela 5 apresenta-se as comparações das médias de QMT e de AHEMD total por sexo, moradia e renda familiar. 
Tabela 5 - Comparação de médias de QMT e AHEMD total.

\begin{tabular}{|c|c|c|}
\hline Variáveis & QMT médio * & AHEMD total médio * \\
\hline \multicolumn{3}{|l|}{ Sexo } \\
\hline Feminino & 4,38 & 12,81 \\
\hline Masculino & 4,06 & 13,00 \\
\hline \multicolumn{3}{|l|}{ Moradia } \\
\hline Casa & 4,14 & 12,41 \\
\hline Apartamento & 4,36 & 13,36 \\
\hline \multicolumn{3}{|l|}{ Renda } \\
\hline Até 2.500 & 4,27 & $11,73^{b}$ \\
\hline 2.500 a 5.000 & 4,38 & $12,13^{b}$ \\
\hline 5.000 ou mais & 4,08 & $15,15^{\mathrm{a}}$ \\
\hline \multicolumn{3}{|c|}{$\begin{array}{l}\text { *Médias seguidas de mesma letra não diferem } \\
\text { estatisticamente (Teste de Mann-Whitney ou Teste de } \\
\text { Kruskal-Wallis) ao nível de } 5 \% \text { de probabilidade. AHEMD- } \\
\text { SR: Affordance in the Home Environment for Motol } \\
\text { Development Self Report, QMT: Quociente motor total. }\end{array}$} \\
\hline
\end{tabular}

Em relação à qualidade do ambiente na promoção de estimulação motora, observa-se (tabela 5) que famílias com renda de $\mathrm{R} \$ 5.000,00$ ou mais apresentam melhor escore total no AHEMD. Portanto, ocorre melhor escore de oportunidades, mas não repercute no melhor desempenho motor total. Pesquisa ${ }^{24}$ realizada em creches públicas na cidade de Piracicaba, SP, a qual analisou o desempenho motor grosso e sua associação com fatores neonatais, familiares e de exposição à creche, de 145 crianças com até três anos de idade, sugere maiorres riscos de atraso motor global naquelas crianças cujas famílias têm menor renda familiar. Em outro estudo, sobre a prevalência de suspeita de atraso no desenvolvimento neuropsicomotor de 1.363 crianças, com 12 meses de idade, nascidas em Pelotas-RS, e seus possíveis determinantes, constatou-se que crianças de mais baixa renda possuíam o dobro de chance de apresentarem suspeita de atraso no desenvolvimento, quando comparadas com as de melhor renda ${ }^{25}$. Esses fatos não foram observados na presente pesquisa, pois não houve diferença significativa entre as médias de desenvolvimento motor total das faixas de renda familiar, o que pode ser explicado devido à maioria das famílias avaliadas possuírem semelhança na renda.

Ao comparar as médias de AHEMD-SR total em cada faixa de renda familiar, observou-se diferenças significativas, indicando que famílias com renda mais elevada proporcionam lares com melhores oportunidades para o desenvolvimento motor. Desta forma, corroborando com este resultado, pesquisa ${ }^{15}$, que avaliou 0 ambiente domiciliar utilizando o AHEMD-IS (Infant scale) de 239 lactentes, com idade entre três e 18 meses, residentes no município de Juiz de Fora, MG, observou que lactentes com melhores níveis econômicos apresentaram oportunidades mais favoráveis ao desenvolvimento motor.

Ainda no que se refere à renda familiar, não houve diferença significativa para os três níveis de renda e o desempenho motor global das crianças. Este resultado leva a refletir sobre as limitações do estudo no que se refere ao grupo específico estudado de crianças matriculadas em IEl particulares.

A tabela 6 remete as correlações obtidas entre o AHEMD-SR e o PMDS-2. Observa-se que ocorreram apenas correlações fracas (correlações próximas de zero) entre as variáveis QMT, QMF, QMG e as variáveis AHEMD total, número de crianças, espaço exterior, espaço interior, variedade de estimulação do AHEMD-SR e materiais de motricidade fina e grossa do PDMS-2. Contudo, apesar de ser fraca, houve correlação significativa entre número de adultos e QMF. 
Tabela 6 - Correlações entre Variáveis do questionário AHEMD-SR e PMDS-2.

\begin{tabular}{c|c|c|c}
\hline Variáveis & QMT & QMF & QMG \\
AHEMDt & 0,11 & 0,06 & 0,13 \\
Espaço exterior & 0,15 & 0,22 & 0,24 \\
Espaço interior & $-0,08$ & $-0,18$ & $-0,11$ \\
Variedade de estimulação & $-0,02$ & $-0,03$ & 0,03 \\
Materiais de motricidade fina & 0,08 & 0,06 & 0,05 \\
Materiais de motricidade grossa & 0,01 & 0,01 & 0,05 \\
Número de adultos & $-0,20$ & $-0,34^{*}$ & $-0,01$ \\
Número de crianças & 0,12 & $-0,03$ & 0,07 \\
\hline${ }^{*}$ Correlação significativa (Correlação de Spearman) ao nível de $5 \%$ de \\
probabilidade. QMF: Quociente motor fino; QMG: quociente motor grosso; QMT: \\
Quociente motor total. AHEMD-SR: Affordance in the Home Environment for \\
Motor Development Self Report.
\end{tabular}

Sabe-se que o ambiente domiciliar é apontado como o fator extrínseco que mais influencia o desenvolvimento infantil, porém no presente estudo, não houve correlação significativa entre o AHEMD total, que remete à qualidade do ambiente em que a criança está inserida, e o QMT, quociente motor total. Pesquisa ${ }^{19}$ corrobora com esse resultado, uma vez que obteve uma correlação baixa e negativa entre o AHEMD total e o Coeficiente Motor Amplo do TDMG 2, teste motor utilizado. Já no estudo ${ }^{6}$, que testou a relação entre o desenvolvimento motor e a qualidade do ambiente domiciliar de 51 crianças com idade de 18 a 42 meses, a correlação foi positiva e moderada.

Ocorreram correlações próximas de zero entre QMT e número de crianças que convivem na mesma residência. Resultado semelhante foi encontrado ao correlacionar o desenvolvimento motor global de 41 lactantes (12 a 24 meses de idade, através do teste motor Bayley Scales of Infant and Toddler Development-III) com o número de crianças do domicílio, não obtendo correlação significativa ${ }^{26}$. Entretanto, na presente pesquisa observou-se correlação significativa, porém fraca, entre a variável número de adultos residentes no mesmo domicílio e a variável QMF, o que difere em parte do resultado encontrado na mesma pesquisa supracitada que não observou correlação significativa para número de adultos com as habilidades motoras finas.

\section{Considerações finais}

De maneira geral, pode-se dizer que em relação ao desenvolvimento motor global, a maioria das crianças avaliadas apresentou resultados dentro do padrão de normalidade para a idade cronológica e o ambiente domiciliar possibilita oportunidades razoáveis para o desenvolvimento motor. Não houve correlação significativa entre ambiente e desenvolvimento motor, o que pode ser explicado pela semelhança entre os resultados de desempenho motor das crianças, bem como pela semelhança da realidade social da maioria delas.

Em específico sobre as habilidades motoras finas e grossas, pelo menos metade das residências oferece condições abaixo do desejado para promover o desenvolvimento nestas habilidades. Observa-se ainda que aquelas famílias que apresentaram maior renda tiveram, como reflexo, maiores oportunidades de estimulação. Contudo, essas maiores oportunidades oferecidas parecem não impactar diretamente no desempenho motor das crianças.

Cabe destacar que alguns aspectos podem ter limitado a capacidade de análise deste estudo, como o número de sujeitos reduzido, devido ao fato da grande perda amostral. Outro fator que merece destaque é a similaridade entre as crianças e os lares investigados. $O$ fato das crianças apresentarem predominância de desenvolvimento motor adequados para a idade e os ambientes domiciliares apresentarem condições muito similares entre si pode dificultar as analises entre elas.

Finalmente, cabe ressaltar a importância de avaliar os outros ambientes em que a criança está inserida ativamente que vá além de sua residência. Avaliação de contextos que fazem parte da sua rede social como a casa dos avós, tios, vizinhos e a própria creche que ele frequenta ajudariam a compreender melhor os efeitos dos múltiplos contextos no desenvolvimento motor infantil. 


\section{Referências bibliográficas}

1. Miranda LP, Resegue R, Figueiras ACM. A criança e o adolescente com problemas do desenvolvimento no ambulatório de pediatria. J. pediatr. 2003;79 supl 1:33-42.

2. Mancini MC, Megale L, Brandão MB, Melo APP, Sampaio RF. Efeito moderador do risco social na relação entre risco biológico e desempenho funcional infantil. Rev. bras. saúde mater. infant. 2004;4(1):25-34.

3. Pilz EML, Schermann LB. Determinantes biológicos e ambientais no desenvolvimento neuropsicomotor em uma amostra de crianças de Canoas/ RS. Ciênc. saúde coletiva. 2007;12(1):181-190.

4. Venetsanou F, Kambas A. Environmental Factors Affecting Preschoolers' Motor Development. Early Childhood Education Journal. 2010;37:319-327.

5. Haydari A, Askari P,Nezhad MZ. Relationship between affordances in the home environment and motor development in children age 18- 42 months. Journal of Social Sciences. 2009;4(5):319- 328.

6. Rodrigues L, Gabbard C. Avaliação das oportunidades de estimulação motora presentes na casa familiar: projecto affordances in the home environment for motor development. Desenvolvimento Motor da Criança. Lisboa: Edições FMH; 2007. p.51-60.

7. Nobre FSS, Pontes ALFN, Costa CLA, Caçola P, Nobre GC, Valentini NC. Affordances em ambientes domésticos e desenvolvimento motor de pré-escolares. Pensar a prática. 2012;15(3):652-668.

8. Freitas TCB. Relação entre as oportunidades de estimulação motora presentes no ambiente domiciliar e a condição socioeconômica da família [dissertação]. Piracicaba: Universidade Metodista de Piracicaba; 2011.

9. Castanho AAG, Assis SMB. Caracterização do Desenvolvimento Motor da Criança Institucionalizada. Fisioter. Bras. 2004;5(6):437-442.

10. Nazario PF, Peres LW, Krebs RJ. A influência do contexto no comportamento motor: Uma revisão. EFDeportes. 2011;15(152). Disponível em: <http://www.efdeportes.com/efd152/a-influencia-do-contexto-no-comportamento-motor. htm>. Acessado em: 5 de fevereiro de 2013.

11. Valentini NC, Rudisill ME. An inclusive Mastery Climate Intervention on the Motor Skill Development of children. APAQ. 2004;21(4):330- 347.

12. Valentini NC, Rudisill ME. Motivational Climate, Motor- Skill Development, and Perceived Competence: two studies of developmentally delayed kindergarten children. J Teach Phys Educ. 2004;23(3):216-234.

13. Fernandes MJ. Estudo Exploratório da Peabody Developmental Motor Scales-2 (PDMS-2), dos 36 aos 71 meses de idade [dissertação]. Lisboa: Universidade Técnica de Lisboa, Faculdade de Motricidade Humana; 2011.

14. Thomas JR, Nelson JK, Silverman SJ. Métodos de pesquisa em atividade física. 6. ed. Porto Alegre: Artmed, 2012.

15. Defilipo, E. C.; Frônoo, J. S.; Teixeira, M. T. B.; Leite, I. C. G.; Bastos, R. R.; Vieira, M. T.; Ribeiro, L. C. Oportunidades do ambiente domiciliar para o desenvolvimento motor. Rev. saúde pública. 2012;46(4):633-41.

16. Folio MR, Fewell RR. Motor Activities Program: PDMS-2 - Peabody Developmental Motor Scales. 2.ed. Texas: Pro-ed, 2000.

17. Teixeira M. (2011) 171 f. Contributo para a validação da versão Portuguesa da PDMS-2. Dissertação de Mestrado, Universidade Católica portuguesa, Lisboa.

18. Siegel S, Castellam Júnior NJ. Estatística não-paramétrica para ciências do comportamento. 2. ed. Porto Alegre: Artmed, 2006.

19. Nobre FSS, Costa CLA, Oliveira DL, Cabral DA, Nobre GC, Caçola PM. Análise das oportunidades para 0 desenvolvimento motor (Affordances) em ambientes domésticos no Ceará, Brasil. RBCDH. 2009;19(1):9- 18.

20. Pilatti I, Haas T, Sachetti A, Fontana C, Oliveira SG, Schiavinato JCC. Oportunidades para o Desenvolvimento Motor Infantil em Ambientes Domésticos. Rev. bras. ciênc. saúde. 2011;9(27):22-27.

21. Müller AB. Efeitos da Intervenção Motora em Diferentes contextos no Desenvolvimento da criança com atraso motor [dissertação]. Porto Alegre: Universidade Federal do Rio Grande do Sul; 2008.

22. Schobert L. O Desenvolvimento Motor de Bebês em Creches: um olhar sobre diferentes contextos [dissertação]. Porto Alegre: Escola de Educação Física da Universidade Federal do Rio Grande do Sul; 2008.

23. Souza SC, Leone C, Takano AO, Moratelli HB. Desenvolvimento de pré-escolares na educação infantil em Cuiabá, Mato Grosso, Brasil. Cad. saúde pública. 2008; 24(8):1917-1926. 
24. Santos DCC, Tolocka RE, Carvalho J, Heringer LRC, Almeida CM, Miquelote AF. Desempenho motor grosso e sua associação com fatores neonatais, familiares e de exposição à creche crianças até três anos de idade. Rev. bras. fisioter. 2009;13(2):173-179.

25. Halpern R, Giugliani ERJ, Victora CG, Barros FC, Horta BL. Fatores de risco para suspeita de atraso no desenvolvimento neuropsicomotor aos 12 meses de vida. J. pediatr. 2000;76(6):421-428.

26. Baltieri, L.; Santos, D. C. C.; Gibim, N. C.; Souza, C. T.; Batistela, A. C. T.; Tolocka, R. E. Desempenho motor de lactentes frequentadores de berçários em creches públicas. Rev. paul. pediatr. 2010;28(3):283-289.

\section{Juliana Falcão Padilha}

Endereço para correspondência - Rua Paula Ramos, 1223. Bairro Coqueiros, CEP 88080-401, Florianópolis, SC, Brasil.

E-mail: jufpadilha@gmail.com

Currículo Lattes: http://lattes.cnpq.br/8493933674992420

Enio Júnior Seidel - ejrseidel@hotmail.com

Fernando Copetti - copettif@gmail.com

Recebido em 24 de setembro de 2013.

Publicado em 22 de maio de 2014. 
